\title{
Note on the orbit of the Great Comet 1882 II.
}

Although the following results can make no pretence to be anything like decisive, still they may be of some slight interest as showing the probable degree of excentricity and the difficulties we may have to deal with in the definitive discussion of the orbit of the Great Comet. The principal source of uncertainty lies in the choice of a point in the disintegrated nucleus which may be considered as the representative of the sharply defined condensation this latter presented before September 30 . At present, and for some time past, it has only been possible to fix the position of the centre of the elongated nebulous mass constituting the nucleus and in what follows the positions refer to as central a point as possible, taking, for instance, the mean of the two nuclei when there were such, and the point halfway between the end ones, when there were more than two.

I make use of the following data, derived from my observations with Dr. Gill's heliometer, kindly placed at my disposal by him:

I) the comparison with $\iota$ Hydrae, Sept. 8 ;

2) the appulse of the conet to the Sun's limb on Sept. I 7, assuming the distance from the Sun's centre to be then $960^{\prime \prime}$ - the Nautical Almanac radius being 957.5 ;

3) a normal place from observations about Sept. 30;

4) a normal place from observations about Oct. 20;

5) a normal place from observations about Nov. 17 ;

The motion is so rapid at 2 ), a variation of the ratio of the curtate distances which produces but 2 " change in 3) and 4) modifying 2) some $\mathbf{8}^{\prime \prime}$, that it is advisable to use it as middle place and I find for the parabola representing exactly 1 ), 2) and 5)

$$
\left.\begin{array}{rl}
T & =\text { Sept. } 17.22380 \text { Greenw. } \\
\log q & =7.887221 \\
w & =69^{\circ} 40^{\circ} 46^{\prime \prime} \\
i & =142 \text { ○ } 14 \\
\delta & =346 \text { 10 } 22
\end{array}\right\} \text { I } 882.0
$$

having the residuals:

$$
\begin{array}{lrr}
\text { Sept. } 30 & \Delta \lambda-3^{\prime \prime}, & \Delta \beta+\mathrm{I}^{\prime} \mathbf{1}^{\prime \prime} \\
\text { Oct. } 20^{\circ} & +14, & +19
\end{array}
$$

from which it appears that the observations cannot be satisfied by a parabola.

Trying then the hypothesis of identity with the 1843 Comet I find for the ellipse through I), 2) and 5) with the semimajor axis $1 \mathrm{I} .6$ corresponding to a period of about 39.5 years

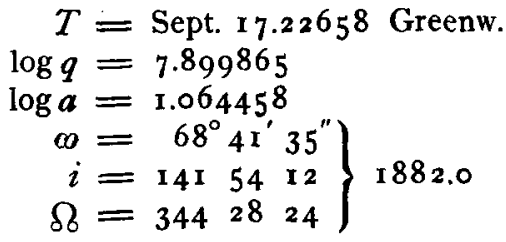

this leaves the residuals

$$
\begin{aligned}
& \text { Sept. } 30 \quad \Delta \lambda+6^{\prime}{ }_{1} 8^{\prime \prime}, \quad \Delta \beta-8^{\prime} 24^{\prime \prime} \\
& \text { Oct. } 20 \quad+426, \quad-920 \text {, }
\end{aligned}
$$

which put this supposition, always somewhat improbable, quite out of the question. They show however that an ellipse with the semimajor axis roo would about destroy the error of the parabola in latitude, and I find for the orbit under this assumption:

$$
\begin{aligned}
& T=\text { Sept. I 7.22394 .Greenw. } \\
& \log q=7.888749 \\
& \log a=2.000000 \\
& \infty=69^{\circ} 34^{\prime} 7^{\prime \prime} \\
& i=1415935\} 1882.0 \\
& 8=3455^{8} 49
\end{aligned}
$$

residuals :

$$
\begin{array}{lrrr}
\text { Sept. } 30 & \Delta \lambda+39^{\prime \prime}, & \Delta \beta+\text { I }^{\prime \prime} \\
\text { Oct. } 20 & +50, & +1 .
\end{array}
$$

A linear interpolation of the elements and the residuals between this orbit and the parabola will be admissible, and shows that, as far as these observations are concerned, the most probable value of $\frac{I}{a}$ is about 0.0075 , an orbit which should represent 3) and 4) as follows:

$$
\begin{array}{lrl}
\text { Sept. } 30 & \Delta \lambda+28^{\prime \prime}, & \Delta \beta+29^{\prime \prime} \\
\text { Oct. } 20 & +41, & +18 .
\end{array}
$$

As errors of the observations these are of course inadmissible and the conclusion appears inevitable that the centre of the nucleus, as deduced by me, is not the point gravitating around the Sun. Unfortunately however the want of observations here from Sept. 8 to Sept. 17 has made it impossible to test the hypothesis of a disturbance at the Comet's passage through the corona which might possibly render it necessary to modify the above conclusions.

Royal Observatory Cape of Good Hope 1882 Dec. I 9 . W. L. Elkin. 